\title{
Low-Impedance 3D PEDOT:PSS Ultramicroelectrodes
}

\author{
Peter D. Jones ${ }^{1 * \dagger}$, Anastasiya Moskalyuk ${ }^{2 \dagger}$, Clemens Barthold ${ }^{1,3}$, Katja Gutöhrlein ${ }^{1,4}$, \\ Gerhard Heusel', Birgit Schröppel', Ramona Samba ${ }^{5 *}$ and Michele Giugliano ${ }^{2,6 *}$ \\ ${ }^{1}$ Natural and Medical Sciences Institute (NMI) at the University of Tübingen, Reutlingen, Germany, ${ }^{2}$ Theoretical Neurobiology \\ \& Neuroengineering, University of Antwerp, Antwerp, Belgium, ${ }^{3}$ joimax GmbH, Karlsruhe, Germany, ${ }^{4}$ Bosch Sensortec \\ $\mathrm{GmbH}$, Reutlingen, Germany, ${ }^{5} \mathrm{NMI}$ Technologietransfer GmbH, Reutlingen, Germany, ${ }^{6}$ Neuroscience Sector, International \\ School for Advanced Studies (SISSA), Trieste, Italy
}

The technology for producing microelectrode arrays (MEAs) has been developing since the 1970s and extracellular electrophysiological recordings have become well established in neuroscience, drug screening and cardiology. MEAs allow monitoring of long-term spiking activity of large ensembles of excitable cells noninvasively with high temporal resolution and mapping its spatial features. However, their inability to register subthreshold potentials, such as intrinsic membrane oscillations and synaptic potentials, has inspired a number of laboratories to search for alternatives to bypass the restrictions and/or increase the sensitivity of microelectrodes. In this study, we present the fabrication and in vitro experimental validation of arrays of PEDOT:PSScoated 3D ultramicroelectrodes, with the best-reported combination of small size and low electrochemical impedance. We observed that this type of microelectrode does not alter neuronal network biological properties, improves the signal quality of extracellular recordings and exhibits higher selectivity toward single unit recordings. With fabrication processes simpler than those reported in the literature for similar electrodes, our technology is a promising tool for study of neuronal networks.

Keywords: ultramicroelectrodes, PEDOT, electrodeposition, neurotechnology, MEA

\section{INTRODUCTION}

Recording and mapping the electrical activity of neurons in vivo, with adequate spatiotemporal resolution to capture both their action potentials and complex synaptic interactions, represents the holy grail of neurotechnology. Disentangling neuronal electrical activity and network communications is in fact a key step towards an in-depth understanding of the nervous system during behavior, sensation, memory, and cognition. Often, advanced technological routes are first explored and validated with reduced in vitro models such as brain tissue slices and dissociated cell cultures. Refinement and technology optimization in vitro is thus pivotal for later translating the prototypes to an in vivo nervous system, and two main electrophysiological approaches were first devised in vitro: (1) intracellular recordings from single neurons, characterized by a high temporal accuracy, selectivity and sensitivity but low spatial resolution while causing irreversible cell damage; and (2) extracellular recordings by microelectrode arrays (MEAs), capable of simultaneously accessing the electrical activity from a large number of cells over long time periods, but lacking sensitivity to subthreshold membrane potentials. Achieving the sensitivity and selectivity of intracellular and patch-clamp techniques from a large number of cells by non-invasive 
means is thus an intense field of research, although definitive technological solutions have yet to be discovered despite promising recent progress (Spira et al., 2018). Further scientific studies, focused on electrical, electrochemical, physical, and physicochemical properties of microelectrodes and cells are therefore urgently needed.

From an electrical perspective, an ideal recording microelectrode would be a zero-area point to increase spatial selectivity while avoiding spatial averaging, with a negligible electrical impedance to minimize thermal noise (Humphrey and Schmidt, 1990; Wellman et al., 2018). An intrinsic benefit of small electrodes is their improved signal fidelity due to reduced spatial averaging (Buitenweg et al., 2002; Viswam et al., 2019). In the context of resolving the activity of single cells, "zero-area" should be subcellular (i.e. much smaller than $10 \mu \mathrm{m}$ ), while nowadays most commercial MEAs have electrode diameters above $10 \mu \mathrm{m}$ and thus do not meet this strict definition. This applies to sparse microelectrode arrays, while smaller electrodes additionally enable increasing the spatial resolution in dense arrays (e.g. CMOS MEAs). However, smaller electrodes require additional care to ensure low electrochemical impedance and thermal noise, and have stricter requirements for shunt capacitance of electrical connections (Robinson, 1968).

Electrodes resolving single-cell activity often measure extracellular action potentials of several nearby cells (e.g., within a range of $150 \mu \mathrm{m}$ ) (Marblestone et al., 2013), so that individual detected signal sources must be disentangled by data analysis and post-processing. Spike sorting is one of such methods and it improves our understanding of network activity by isolating the spike trains of putative single cells, although requiring higher sampling rates (e.g., $25 \mathrm{kHz}$ ) to resolve spike shapes, thus increasing the amount of data to be stored and analyzed. In this respect, realtime data interpretation, required in some "closed-loop" applications (e.g., neuroprosthetics), is more challenging and long recording sessions encounter practical limitations in terms of data storage (Navajas et al., 2014). Recent results with small-sized microelectrodes have demonstrated how an improved electrical coupling of cell membranes to the microelectrodes could significantly decrease the distance between the membrane and the electrode surface and thus increase the effective sealing resistance at the interface, improving the selectivity and in principle removing the need for spike sorting (Ojovan et al., 2015).

Even when single cells are isolated, extracellular recordings can hardly discriminate between action potentials of excitatory and inhibitory neurons. Statistical metric-based approaches to distinguish excitatory from inhibitory neurons had been already proposed (Becchetti et al., 2012), but combinations of spike waveforms and timing parameters from extracellular recordings do not easily allow distinguishing GABAergic interneurons from non-GABAergic neurons in vitro from their electrophysiological signature alone (Weir et al., 2014). Recently, a CMOS-based array of 4096 nanoelectrodes was reported to be capable of identifying excitatory and inhibitory synaptic connections by means of intracellular recordings, achieved by electroporation of neuronal membranes (Abbott et al., 2019).
In fact, it is today well accepted that microelectrodes are not passive "observers". Their shape and surface chemical properties can enable recognition by cells and alter the electrical coupling between the cell membrane and the electrode. Nonetheless, an ideal and robust strategy to fully access intracellular electrical potentials by extracellular microelectrodes has not yet been discovered. Vertical nanowires (Almquist and Melosh, 2010; Robinson et al., 2012; Wesche et al., 2012; Xie et al., 2012; Angle et al., 2014; Lin et al., 2014; Qing et al., 2014; Dipalo et al., 2017) or microscale mushroom-shaped electrodes (Hai et al., 2010b; Santoro et al., 2013) have most widely been used to improve cell-electrode coupling, up to the point of revealing intracellular signals (Spira et al., 2018). Nanowires are intended to discretely penetrate the cell membrane, while "mushrooms" may mimic the shape of dendritic spines and encourage close contact by natural membrane-engulfment processes. Both methods were shown to achieve attenuated intracellular-like recordings, demonstrating signal-to-noise ratios, signal amplitude, and invasiveness on a spectrum between classical extracellular and intracellular recordings (Guo, 2019). Most of the promising results in the recent literature rely on active perforation of cell membranes (e.g., by electrical or optical means) to temporarily expose the microelectrode to the cell's cytosol and thus to the intracellular electrical potential, even with the risk of non-recoverable cellular damage. Biological functionalization of the microelectrode surface has also been demonstrated to be beneficial (Hai et al., 2010a), so that the active recruitment of ion channels to the junctional area of the membrane might explain the spontaneous (i.e., unstimulated) intracellular-like signals (Shmoel et al., 2016). A small proportion of intracellularlike recordings with gold mushroom-shaped microelectrodes displayed unusually large peak-to-peak amplitudes for neuronal signals, above $5 \mathrm{mV}$, which were hypothesized to result from the spontaneous relocation of passive ion channels to the part of the membrane facing the electrode, thus effectively lowering the junctional membrane impedance (Shmoel et al., 2016). Promising results of mushroom-shaped electrodes have benefited from the stability and inertness of gold microstructures and their ease of use in microfabrication, despite suffering from its high electrochemical impedance. We argue below that the spike amplitudes above $5 \mathrm{mV}$ reported by Shmoel et al. (2016) could even have been attenuated by capacitive shunting phenomena and would have revealed even stronger signal amplitudes if the microelectrodes had considerably lower impedances. Fully understanding the mechanisms underlying such unusually strong recordings is a promising path to engineering reliable MEA-based intracellular recordings, ultimately enabling stable, long-term future measurements.

In this paper, we describe and experimentally validate in vitro the use of conductive polymer coating on $3 \mathrm{D}$ ultramicroelectrodes, fabricated with a near-ideal size of $2 \mu \mathrm{m}$. The 3D structure of our electrodes increases surface area while maintaining minimal lateral size, and their coating with poly(3,4-ethylenedioxythiophene):polystyrene sulfonate (PEDOT:PSS) reduced the electrochemical impedance, achieving acceptable thermal noise levels. We ultimately successfully achieved electrode dimensions down to $1 \mu \mathrm{m}$ by means 
of optical lithography, thus demonstrating an economical fabrication route in contrast to similar results relying on electron beam lithography (Weidlich et al., 2017). In comparison to CMOS MEAs (Abbott et al., 2019), our MEAs are fabricated on transparent glass substrates, allowing the easy combination with transmitted light microscopy. While PEDOT coatings previously decreased the impedance of 15 - $\mu$ m-large microelectrodes (Ludwig et al., 2011), here we demonstrate for the first time how PEDOT:PSS coating of 3D ultramicroelectrodes allows a similar final result. Preliminary data from this work were presented at a conference (Jones et al., 2016). Here, we detail a robust production process for serial fabrication of in vitro probes, and experimentally validate their operation, stability, and suitability for neural electrophysiology.

\section{MATERIALS AND METHODS}

\section{Fabrication}

Fabrication of microelectrode arrays (MEAs) for in vitro applications, featuring PEDOT:PSS-coated ultramicroelectrodes, was achieved by adapting the technological processes employed for standard MEA fabrication. The key steps are illustrated in Figure 1.

Briefly, MEAs with a large conventional internal reference electrode and 59 ultramicroelectrodes, arranged in an $8 \times 8$ square grid with a pitch of $200 \mu \mathrm{m}$, were fabricated on 1-mmthick, $49 \times 49 \mathrm{~mm}^{2}$ borosilicate float glass substrates. Conducting

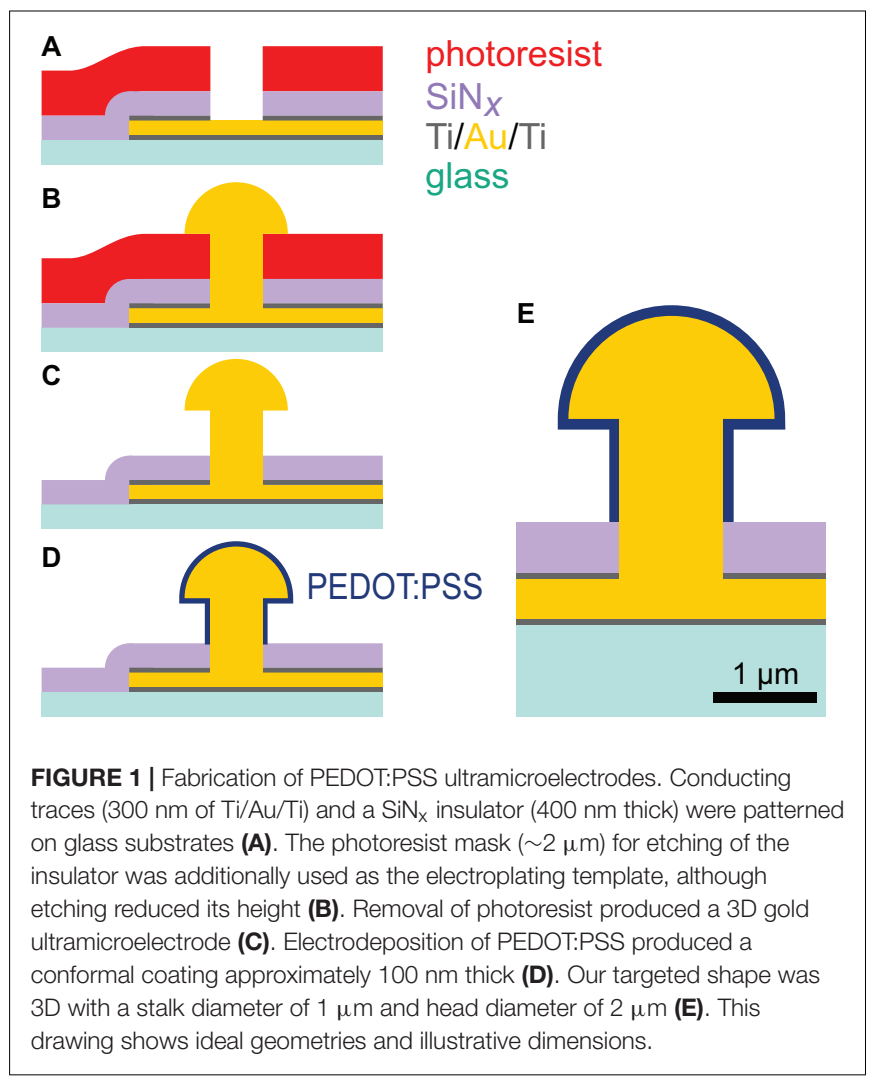

traces were produced by sputtering $300 \mathrm{~nm}$ of titanium and gold (Z550, Leybold, Cologne, Germany), defining an S1818 etch mask by photolithography (Gamma, SÜSS MicroTec SE, Garching, Germany), and plasma etching ( $\mathrm{CF}_{4}$ and Ar, Z550). Silicon nitride $\left(\mathrm{SiN}_{\mathrm{X}}, \sim 400 \mathrm{~nm}\right.$ ) was deposited by PECVD (Plasmalab 800, Oxford Instruments, Abingdon, United Kingdom) for insulation. The insulator and upper Ti layer were etched with $\mathrm{CF}_{4}$ and Ar plasma (Plasmalab 800) with a $\sim 2 \mu \mathrm{m} \mathrm{AZ} \mathrm{ECI}$ 3027 resist mask (developed in diluted AZ 351B), which further served as a template for electrodeposition (with reduced height due to non-ideal etch selectivity). Achieving $1 \mu \mathrm{m}$ feature sizes required advanced optimization of the lithography parameters. Photomasks with $0.75 \mu \mathrm{m}$ features were used to obtain diameters down to $1 \mu \mathrm{m}$.

Before electroplating, microelectrodes were electrochemically cleaned by voltage cycling in sulfuric acid $\left(0.5 \mathrm{M} \mathrm{H}_{2} \mathrm{SO}_{4}\right.$, 0.4-1.4 V vs. $\mathrm{Ag} / \mathrm{AgCl}$, scan rate $300 \mathrm{mV} / \mathrm{s}$ ). In this step, cyclic voltammograms were measured for individual electrodes to confirm whether etching had exposed gold. 3D gold ultramicroelectrodes were individually electroplated (VMP3 multichannel potentiostat, Bio-Logic SAS, Seyssinet-Pariset, France) from a gold electrolyte (NB Semiplate Au 100, NB Technologies $\mathrm{GmbH}$, Bremen, Germany) heated to $40^{\circ} \mathrm{C}$. A current of $-0.4 \mathrm{nA}$ was applied and the plating time was adapted according to the desired electrode size. Typical dimensions of $1 \mu \mathrm{m}$ stalk height, $1 \mu \mathrm{m}$ stalk diameter, and 1.5$2.0 \mu \mathrm{m}$ cap diameter required $\sim 85 \mathrm{~s}$. After electrodeposition, photoresist was removed by stirring substrates in $0.5 \mathrm{M} \mathrm{KOH}$ at $50^{\circ} \mathrm{C}$ for $5 \mathrm{~min}$.

The gold ultramicroelectrodes were coated with PEDOT:PSS via electrodeposition (Gerwig et al., 2012). Prior to PEDOT:PSS coating, MEAs were treated with a $100 \mathrm{~W}$ air plasma for 2 min (Harrick plasma cleaner, Ithaca, NY, United States) and microelectrodes were again cleaned by voltage cycling in sulfuric acid; here, the voltage was applied simultaneously to all electrodes. The electrodes were rinsed then immersed in an aqueous solution of $1 \%$ PSS with $0.03 \mathrm{M}$ 3,4ethylenedioxythiophene (EDOT). A constant current of $0.2 \mathrm{nA}$ $\left(\sim 0.02 \mathrm{nA} / \mu \mathrm{m}^{2}\right)$ was applied for various time intervals, lasting up to $30 \mathrm{~s}$, to deposit charges of up to $6 \mathrm{nC}$.

\section{Electrochemical Characterization}

Measurements were made with a Pt mesh counter electrode in phosphate-buffered saline (PBS). Impedance spectra were acquired from $1 \mathrm{~Hz}$ to $100 \mathrm{kHz}$ with an amplitude of $10 \mathrm{mV}$. The electrode thermal noise was measured with a ME2100 amplifier (Multi Channel Systems MCS GmbH, Germany).

\section{Optical and Scanning Electron Microscopy}

Optical images were acquired with an upright Olympus microscope and a 100x objective lens. Focused-ion beam (FIB) milling and scanning electron microscopy (SEM) were performed with a Zeiss AURIGA CrossBeam. Samples were sputtered with a thin layer of AuPd for conductivity prior to SEM. FIB crosssections revealed precise $3 \mathrm{D}$ and internal structures. 


\section{Stability}

MEAs were stressed while their stability was monitored by optical microscopy and impedance measurements. Stresses included sonication in an ultrasound bath for $30 \mathrm{~s}$, treatment with air plasma for $30 \mathrm{~s}$, incubation in $96 \%$ ethanol for $14 \mathrm{~h}$ and submersion in $\mathrm{PBS}$ at $37^{\circ} \mathrm{C}$.

\section{CELL CULTURES, ELECTROPHYSIOLOGY, AND DATA ANALYSIS}

\section{Dissociated Cell Cultures}

The experiments were carried out in compliance with the European Community Council directive and approved by the Animal Ethics Committee of the University of Antwerp. Prior to cell seeding, all MEAs were treated with air plasma $(\sim 10 \mathrm{~W}$ for $90 \mathrm{~s}$ ) in a plasma cleaner (Zepto BRS, Diener electronic $\mathrm{GmbH}$, Ebhausen, Germany), sterilized with $70 \%$ ethanol for $30 \mathrm{~min}$, rinsed in Milli-Q water for $30 \mathrm{~min}$, covered with polyethyleneimine at room temperature overnight (PEI, 0.1\% wt/vol in Milli-Q water, Sigma-Aldrich, Germany), then rinsed with Milli-Q water and air-dried for $30 \mathrm{~min}$. Neuronal cultures were prepared from cortical or hippocampal neurons from newborn rats using standard procedures. Briefly, the skull was removed, and the brain isolated. The brain area was dissected out and cut into small pieces which were incubated for $15 \mathrm{~min}$ at $37^{\circ} \mathrm{C}$ in $0.025 \%$ trypsin (Sigma-Aldrich, Germany) solution. After enzymatic treatment the tissues were washed and mechanically dissociated. The cell suspension was diluted in order to control final density of seeding and were plated on MEAs with initial density of 6500 cells $/ \mathrm{mm}^{2}$. Cells were maintained in minimum essential medium (MEM), containing 10\% horse serum, and cultured in a $5 \% \mathrm{CO}_{2}$ incubator at $37^{\circ} \mathrm{C}$ and $95 \%$ humidity. At DIV 3 cells were incubated with $1 \mu \mathrm{M}$ arabinosylcytosine for 24 h. Every 2 days half of the culture medium was replaced with fresh, pre-warmed medium and, starting from DIV12 onwards, the replacing of culture medium was performed with serum free medium.

\section{Extracellular Electrophysiology and Spike Sorting}

Arrays of PEDOT-coated ultramicroelectrodes were tested in comparison to standard commercial MEAs (60MEA200/30iRITO-gr, Multi Channel Systems MCS GmbH, Reutlingen, Germany) containing 60 titanium nitride (TiN) planar microelectrodes, each with a diameter of $30 \mu \mathrm{m}$ and with the same $8 \times 8$ layout and $200 \mu \mathrm{m}$ pitch. We carried out recordings of the spontaneous neuronal activity at $37^{\circ} \mathrm{C}$ and $5 \%$ $\mathrm{CO}_{2}$ for at least $30 \mathrm{~min}$, starting from 7 and continuing up to 35 days in vitro. For each electrode the extracellular voltage was detected and amplified by a MEA-1060-Up-BC multichannel amplifier, with a $1-3000 \mathrm{~Hz}$ bandwidth and an amplification factor of 1200 (MCS GmbH). Raw analog signals were acquired with a sampling frequency of $25 \mathrm{kHz}$ and converted to digital signals at a resolution of 16 bits, using an A/D converter
(MCCard, MCS GmbH), leading to an optimal representation of the spike waveform. Slow components and high frequency noise of the digital signals were filtered out by a band-pass filter, between 300 and $3000 \mathrm{~Hz}$. Filtered signals were stored on a disk for offline data processing. Action potential detection was performed with QSpike Tools (Mahmud et al., 2014) using for each channel an individual threshold, automatically calculated as five times the standard deviation of the "background" noise (Quiroga et al., 2004). Each recorded channel with a detected neuronal spiking activity higher than $0.5 \mathrm{~Hz}$ was considered as an "active" electrode. All spike waveforms were aligned to the peak and represented by 64 data points (i.e., $2.56 \mathrm{~ms}$ ). Spike times and spike waveforms were stored on disk for subsequent analyses, performed by custom scripts in MATLAB (The MathWorks, Natick, MA, United States). Dimensionality reduction was done with wavelet transforms by extracting distinctive features from the detected spike waveforms (Quiroga et al., 2004). This time-frequency decomposition of the signals was performed by using Haar wavelets. The selection of the best separating wavelet coefficients was done automatically, using a KolmogorovSmirnov test for normality, and the less normally distributed wavelet coefficients were chosen. For spike sorting we used the superparamagnetic clustering procedure (Quiroga et al., 2004). Briefly, this unsupervised method allows to group the chosen wavelet coefficients into clusters, using only one parameter, called "clustering temperature." In case of "low" temperature, all data is grouped into one cluster, while increasing temperature leads to split of the dataset into many clusters. An optimal middle range or "superparamagnetic" temperature exists, when the data is assigned to few large clusters. After this step, spikes with the different shapes were grouped into subsets, corresponding to the isolated neuronal units.

\section{Statistics}

Normality of the distribution was verified by the Lilliefors test. For normal distributions data are shown as the mean \pm standard error of the mean (SEM); statistical significance between groups is assessed using a two-way ANOVA and post-hoc Fisher's procedure. Values $p<0.05\left(^{*}\right)$ and $p<0.01\left(^{* *}\right)$ were considered as significant differences.

\section{RESULTS}

\section{Fabrication}

We successfully fabricated arrays of 59 PEDOT:PSS-coated, mushroom-shaped gold ultramicroelectrodes. Small sizes of ultramicroelectrodes are known to be critical for a tight contact with cells' membranes (Ojovan et al., 2015). By photolithography and electroplating, our ultramicroelectrode stems had diameters as small as $1 \mu \mathrm{m}$. These dimensions approach the smallest resolvable feature size for contact lithography, which is on the order of $W_{\text {min }} \approx \sqrt{\lambda d}$ for wavelength $\lambda$ and mask-substrate distance $d$ (Plummer et al., 2000). We predicted $W_{\min } \approx 950 \mathrm{~nm}$ with a resist thickness of $2 \mu \mathrm{m}$ and unfiltered emission from a Hg lamp of 300 to $450 \mathrm{~nm}$. Approaching this limit required 
photomask feature sizes of $750 \mathrm{~nm}$, and optimization of spincoating, exposure, and development processes. Etching of the insulator was optimized to reveal the gold traces while avoiding overetching that would enlarge ultramicroelectrode diameters.

Cyclic voltammograms in $\mathrm{H}_{2} \mathrm{SO}_{4}$ not only cleaned the gold ultramicroelectrodes but also allowed us to identify if plasma etching had successfully exposed the gold. The peak at $\sim 0.6 \mathrm{~V}$ versus $\mathrm{Ag} / \mathrm{AgCl}$ due to reduction of chemisorbed oxygen reflects the gold surface area, with charge for polycrystalline gold of $3.9 \mathrm{pC} / \mu \mathrm{m}^{2}$ (Trasatti and Petrii, 1992). The area of gold exposed prior to gold electroplating was $<1 \mu \mathrm{m}^{2}$. Residual $\mathrm{Ti}$ at the perimeter of the gold area affected the specific area (as revealed by FIB cross-sections).

The caps of each ultramicroelectrode could be precisely controlled in size by current-controlled electroplating. Electrodeposition parameters and temperature critically affected the structure of the electrodes. For example, gold deposition with $-1.5 \mathrm{nA}$ produced ultramicroelectrodes with voids in their stems, as revealed by FIB cross-sections. In many cases, such ultramicroelectrodes remained stable through fabrication, even with these invisible voids. Nonetheless, ensuring void-free electrodes was important to us in order to improve the device stability for biological applications. Best results without voids were obtained when using currents of $-0.4 \mathrm{nA}$.

Conformal PEDOT:PSS coatings were produced by adapting established methods (Gerwig et al., 2012). After attempting electrodeposition of composites of PEDOT:PSS with carbon nanotubes (CNT) as reported by Gerwig et al. (2012) we observed uncontrolled formation of PEDOT:PSS/CNT structures extending tens of micrometers away from the gold electrodes. We assumed that this effect was caused by adsorption of CNTs on the $\mathrm{SiN}_{\mathrm{X}}$ insulator. We therefore used PEDOT:PSS without CNTs.

\section{Electrochemical Characterization}

Impedance measurements revealed as expected low impedances of the PEDOT:PSS-coated ultramicroelectrodes, in consideration of their size (Ludwig et al., 2011; Gerwig et al., 2012). Impedances decreased with increasing PEDOT:PSS deposition (Figures 2A,B), and were $442 \pm 86 \mathrm{k} \Omega$ at $1 \mathrm{kHz}(n=105)$ with $6 \mathrm{nC}$ of PEDOT:PSS. Consistent with these values, the median electrode noise was $6 \mu \mathrm{V}_{r m s}$. Approximately $90 \%$ of electrodes were functional (here, 105/118 from two typical MEAs), based on the impedance magnitude below $1 \mathrm{M} \Omega$ at $1 \mathrm{kHz}$. Process optimization would be needed to reach $100 \%$ yield expected for commercial-grade MEAs.

For ultramicro- or nanoelectrodes, spreading resistance in the electrolyte and shunt impedance of conducting traces become increasingly important. Spreading resistance $\left(R_{S} \approx\right.$ $\rho / 2 d$ ) depends on electrode diameter $d$ and medium conductivity $\rho$ (Franks et al., 2005). Here, we estimate that our measurements with PBS $(0.7 \Omega \mathrm{m})$ include spreading resistance on the order of $100 \mathrm{k} \Omega$. While this resistance will appear in impedance measurements, the presence of cells necessarily changes the situation. For example, proximal or engulfing cells will modify the spreading resistance. In this situation, it is more prudent to discuss the seal resistance, which can dramatically increase the magnitude of recorded extracellular potentials (Spira et al., 2018).

We estimate that our shunt capacitances were on the order of $100 \mathrm{pF}$, based on the area of the electrical trace $\left(0.6-0.8 \mathrm{~mm}^{2}\right)$, thickness of the silicon nitride insulator $(400 \mathrm{~nm})$ and its electric permittivity of 7 (Piccirillo and Gobbi, 1990), while the internal capacitance of our potentiostat adds another $10 \mathrm{pF}$. The shunt impedance is in parallel with the electrode and will shunt signals to ground (Robinson, 1968). The shunt capacitance produces a low pass filter, causing attenuation and distortion of higher frequency electrode signals both for recording and stimulation. For a more intuitive comparison, a $100 \mathrm{pF}$ shunt has an impedance magnitude of $1.6 \mathrm{M} \Omega$ at $1 \mathrm{kHz}$.

Impedance spectra over the frequency range most relevant for extracellular electrophysiology $(1-5000 \mathrm{~Hz})$ had phase near $-90^{\circ}$, showing predominantly capacitive behavior. The equivalent capacitances of these electrodes increased to ca. $400 \mathrm{pF}$ with increasing amounts of PEDOT:PSS (Figure 2C). With an estimated surface area of $\sim 20 \mu \mathrm{m}^{2}$, these capacitances are on the order of $2 \mathrm{mF} / \mathrm{cm}^{2}$, similar to those reported by Gerwig et al. (2012). Considering a thickness on the order of $100 \mathrm{~nm}$, these results agree with volumetric capacitance of $100 \mathrm{~F} / \mathrm{cm}^{3}$ calculated for PEDOT:PSS by density functional theory (Sahalianov et al., 2019).

\section{Optical and Scanning Electron Microscopy}

Optical microscopy indicated whether a ultramicroelectrode was bare gold or coated with PEDOT:PSS (Figure 3A), but could otherwise not reveal details. SEM revealed the dimensions and topography of the gold and PEDOT:PSS-coated electrodes (Figure 3). Our gold surfaces were rougher than other gold mushroom-shaped electrodes (Hai et al., 2009). This was caused in part by the $40^{\circ} \mathrm{C}$ temperature during electroplating; higher temperatures produced smoother surfaces but caused early delamination of our photoresist sacrificial layer. Cross-sections produced by FIB milling were necessary to reveal internal $3 \mathrm{D}$ structures, such as the thickness of the PEDOT:PSS layer or internal defects. The thickness of PEDOT:PSS was on the order of $100 \mathrm{~nm}$.

\section{Stability}

Optical microscopy showed no damage to the electrodes after $30 \mathrm{~s}$ of sonication. Impedance spectroscopy showed negligible differences in impedance after $14 \mathrm{~h}$ in ethanol or $30 \mathrm{~s}$ of air plasma, while submersion in $\mathrm{PBS}$ at $37^{\circ} \mathrm{C}$ increased impedance after two weeks (Figure 2D).

\section{Experimental Validation in vitro}

The electrical impedance is an essential characteristic determining the sensitivity of microelectrodes employed for neuronal signal detection. For this reason, we began our investigation by testing the efficiency of neuronal signal recordings while taking into account that the impedance of ultramicroelectrodes is significantly higher than that 


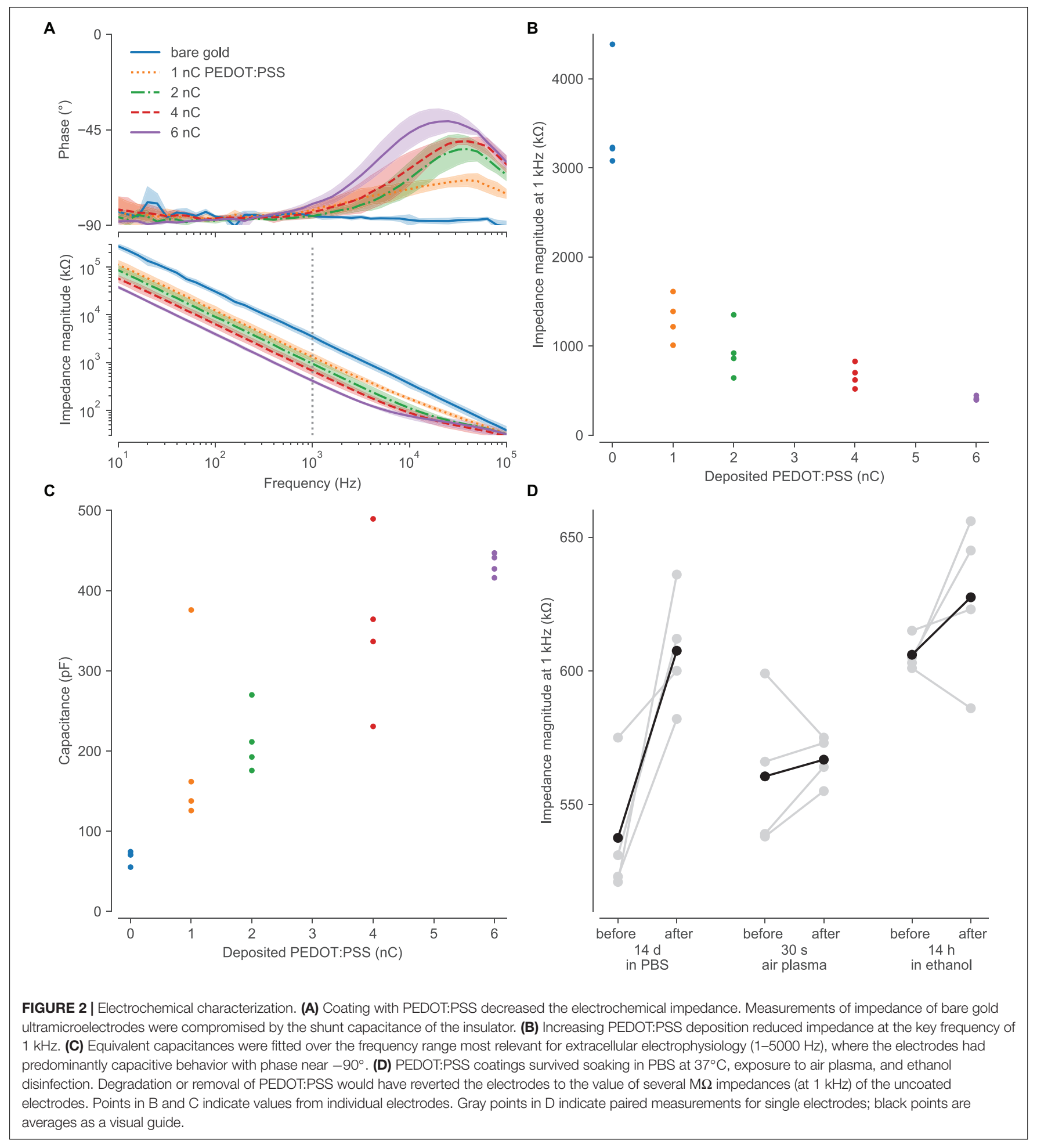

of conventional larger planar electrodes. We tested 10 different rat neuronal cultures (see the section "Materials and Methods") as we analyzed the recordings of spontaneous electrophysiological activity detected by ultramicroelectrodes (Figure 4A, right). As a control, we employed data obtained from the sister cultures, plated on commercial conventional
MEAs (30 $\mu \mathrm{m}$ planar TiN; Figure 4A, left). As expected from ex vivo developing neuronal cell cultures, in all MEAs the electrical neuronal activity was characterized by sparse isolated spikes, starting from 7 days in vitro (DIV7). Spikes became spatially organized into episodic synchronous bursts, starting from DIV10 onwards (Figure 4A, white traces), 


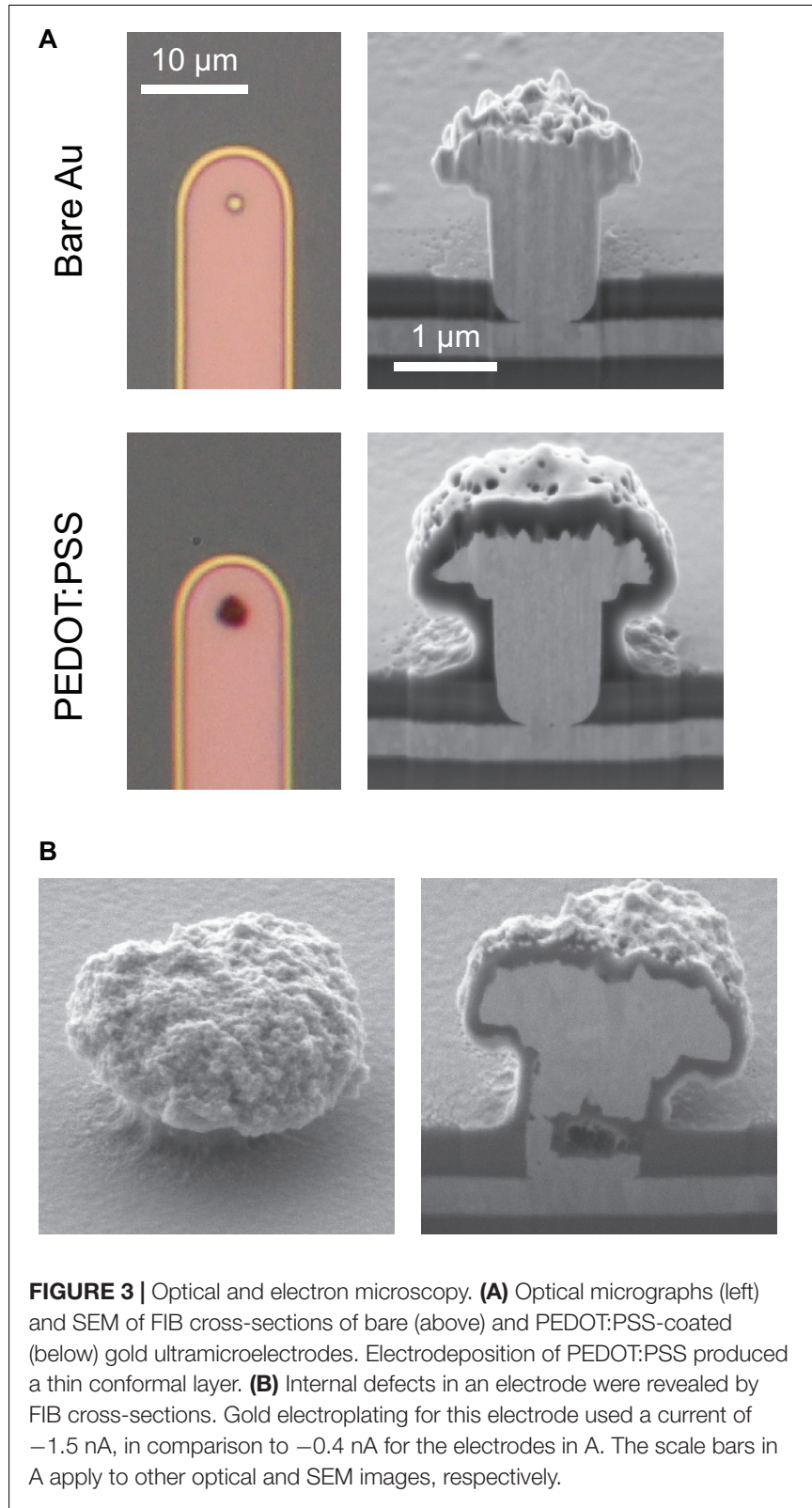

suggesting a normal ex vivo synaptic and network development (Mahmud et al., 2014).

Our data revealed that less than $10 \%$ of (uncoated) gold ultramicroelectrodes were active (see the section "Materials and Methods"), detecting neuronal spontaneous signals (Figure 4B) during an observation interval of $30 \mathrm{~min}$. We also observed that the number of spikes detected per active gold ultramicroelectrodes was $65 \pm 13$ (Figure 4C), significantly $(p<0.01)$ lower than the percentage of active electrodes $(95 \pm 2 \%)$ and number of spikes (1096 \pm 268$)$ detected by conventional planar microelectrodes, over the same time interval.

As soon as the coating with PEDOT:PSS was employed in our ultramicroelectrodes, it greatly improved the sensitivity of the ultramicroelectrodes, so that the fraction of active electrodes significantly $(p<0.01)$ increased and reached $53 \pm 6 \%$
A
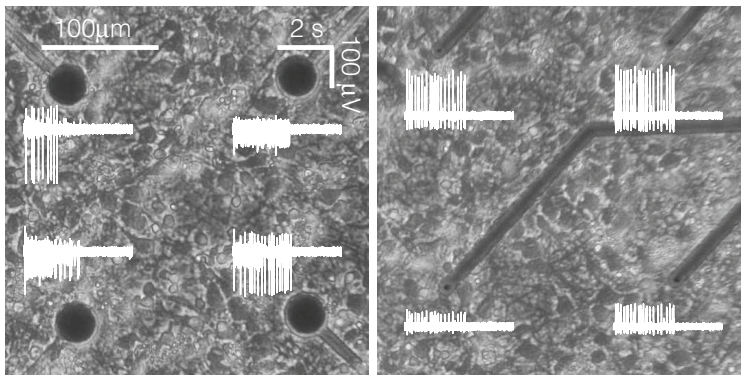

B

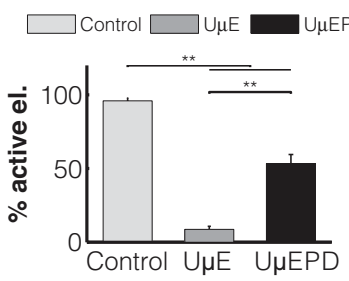

C

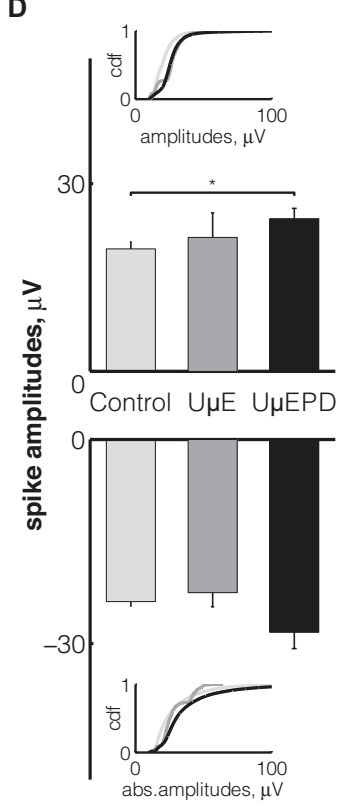

FIGURE 4 | Elementary properties of different types of electrodes:

conventional planar (light gray, $n=12 \mathrm{MEAs})$, gold ultramicroelectrodes $(\mathrm{U} \mu \mathrm{E}$,

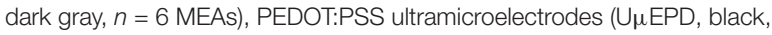
$n=10 \mathrm{MEAs}$ ). Representative phase-contrast micrographs show cortical cells at the second week after plating on the glass surface with conventional planar electrodes (A, left) and with PEDOT:PSS ultramicroelectrodes (A, right). The white traces represent the electrical activity detected by the corresponding electrodes within an interval of $4 \mathrm{~s}$. The percentage of active electrodes is represented as percentage of those electrodes detecting signals (B). The average number of spikes per active electrode is displayed in (C), together with the average value of detected amplitudes with positive (top) and negative (bottom) deflections (D). The average number of different spike waveforms (clusters) detected by single electrode is displayed in panel (E). Data are shown as the mean \pm SEM and statistical significance between groups was assessed using a two-way ANOVA and post-hoc Fisher's procedure.

(Figure 4B), and so that the number of detected spikes by coated electrodes increased to $823 \pm 261$ (Figure 4C), making the differences with conventional electrodes non-significant, over the same time interval.

Regardless of the type of microelectrodes, spike waveforms with positive and negative peak deflections were detected. Their amplitudes varied over a wide rage, although in $50 \%$ cases they were below $35 \mu \mathrm{V}$. The largest amplitudes 
(i.e., up to $\sim 550 \mu \mathrm{V}$ ) were recorded with PEDOT:PSS ultramicroelectrodes. On average, positive $(24 \pm 2 \mu \mathrm{V})$ and negative $(-28 \pm 2 \mu \mathrm{V})$ spike peak amplitudes recorded with PEDOT:PSS ultramicroelectrodes were higher compared to those recorded by conventional planar electrodes $(19 \pm 1 \mu \mathrm{V}$ and $-24 \pm 1 \mu \mathrm{V})$. Interestingly, for positive spike waveform amplitudes this difference was significant $(p<0.05)$ (Figure 4D). Moreover, the cumulative distribution function (Figure 4D, insets) of the absolute values of peak amplitudes detected by PEDOT:PSS ultramicroelectrodes was significantly shifted to the right, i.e., towards higher peak amplitudes, confirming the higher probability to detect higher spike amplitudes by PEDOT:PSS ultramicroelectrodes than by conventional MEAs.

During extracellular recordings by conventional techniques (MEAs), each electrode picks up signals from its spatial proximity and possibly from several neurons simultaneously. The diversity of spike peak amplitudes and waveform shapes requires spike sorting to identify the putative signal sources. Yet, the variability of intracellular action potentials (e.g., amplitude reduction of successive spikes during bursting) is also reflected in the extracellular signals produced by individual neurons, and this must be considered during spike sorting. In order to determine how many distinct neuronal units participated in spike generation during our extracellular recordings, we extracted specific features of the shape of the spikes, so that spike events with similar features were classified as belonging to the same group or "cluster", following a state-of-the-art unsupervised spike sorting technique. After this procedure, each cluster became associated with a single putative isolated neuronal unit. Therefore, by using spike sorting and a well known clustering algorithm (see the section "Materials and Methods"), the spikes detected by planar MEAs, were routinely grouped on average into $2.0 \pm 0.1$ different clusters (i.e., neuronal units) per microelectrode, whereas those detected by gold ultramicroelectrodes were grouped in a significantly $(p<0.01)$ lower number of spike clusters $(1.1 \pm 0.0)$. This result was replicated also in the case of PEDOT:PSS ultramicroelectrodes $(1.2 \pm 0.1)$ (Figure $4 \mathrm{E}$ ), suggesting that the smaller physical dimension of the ultramicroelectrodes determined an increase in the spatial selectivity of spike detection, for each electrode.

One more necessary requirement for microelectrodes is the long-time stability of recordings. We tested our PEDOT:PSS ultramicroelectrodes and evaluated their noninvasive properties vs. planar control microelectrodes, by performing long-term recordings (i.e., over successive days) during the development of network activity in vitro. As for planar electrodes of conventional MEAs, PEDOT:PSS ultramicroelectrodes detected both isolated asynchronous spikes as well as episodic synchronized burst of spikes, over 3 weeks of culturing in vitro (Figure 5A, left panel). During this time, the shape of detected spikes remained stable (Figure 5A, right panel). Synchronized activity across whole neural network was detected for all tested PEDOT:PSS ultramicroelectrode MEAs $(n=10)$ and a typical raster plot (Figure 5B top), integrating network firing rates (Figure 5B middle) and raw voltage traces (Figure 5B bottom), revealed the dynamics of functional networks in vitro, similar to conventional planar electrodes. Long-term monitoring of neuronal network activity throughout the second, the third and the fourth weeks in vitro revealed that both positive (Figure 5C top, conventional planar: $18 \pm 1 \mu \mathrm{V} ; 19 \pm 1 \mu \mathrm{V} ; 19 \pm 1 \mu \mathrm{V}$; PEDOT:PSS ultramicroelectrodes $27 \pm 4 \mu \mathrm{V} ; 24 \pm 2 \mu \mathrm{V}$; $23 \pm 2 \mu \mathrm{V}$ ) and negative (Figure 5C bottom, conventional planar: $-22 \pm 1 \mu \mathrm{V} ;-24 \pm 1 \mu \mathrm{V} ;-23 \pm 1 \mu \mathrm{V}$; PEDOT:PSS ultramicroelectrodes: $-26 \pm 3 \mu \mathrm{V} ;-28 \pm 2 \mu \mathrm{V} ;-28 \pm 2 \mu \mathrm{V})$ peak amplitudes did not change. Despite the amplitudes recorded with PEDOT:PSS ultramicroelectrodes being slightly higher than the ones detected with planar electrodes, a significant difference $(p<0.05)$ was observed only for positive peak amplitude spikes, during the second and third weeks in vitro.

The majority of spike waveforms detected with PEDOT:PSS ultramicroelectrodes had single negative (68\%) or single positive (9\%) peak deflections (Figure 5D, right), whereas only $21 \%$ of conventional planar electrode detected single spikes waveforms with negative peak deflection (Figure 5D, left). The majority of planar electrodes instead detected spikes with biphasic (positive and negative) deflections (65\%) simultaneously, with single positive peak spikes never detected by planar electrodes.

\section{DISCUSSION}

In this work, we aimed to demonstrate the possibility of novel MEA fabrication with low impedance ultramicroelectrodes, exhibiting in comparison to standard MEAs an adequate signalto-noise ratio, an improved selectivity for single units and a proper stability throughout long-term recordings.

The majority of previous studies in the literature suggest that electrode impedance has an important impact on signal-to-noise ratio (Ludwig et al., 2011; Scott et al., 2012; Chung et al., 2015) and therefore on sensitivity to detect spikes, although the opposite point of view has been expressed (Neto et al., 2018). Our data demonstrate that the electrode impedance is crucial for spike detection in extracellular recordings and that a simple and reproducible technique, such as PEDOT:PSS coating, leads to a stable, biocompatible, and significantly increased effectiveness of neural signal recording.

Notably, in the absence of the coating, gold ultramicroelectrodes per se have an expected impedance magnitudes above $10 \mathrm{M} \Omega$ at $1 \mathrm{kHz}$, as estimated by gold's double-layer capacitance of $\sim 0.7 \mathrm{pF} / \mu \mathrm{m}^{2}$ (Piela and Wrona, 1995). In this circumstance, the lower shunt impedance of the insulator prevented an independent measurement of the impedance of gold ultramicroelectrodes. More importantly, recording or stimulation with such uncoated ultramicroelectrodes is severely attenuated due to substantial shunting of the signals to the grounded bath. 
A

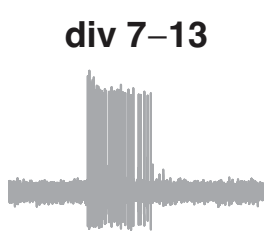

div 14-20

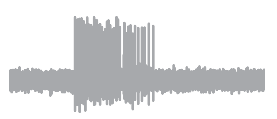

div 21-28

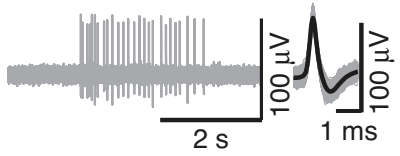

B

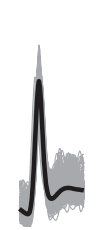

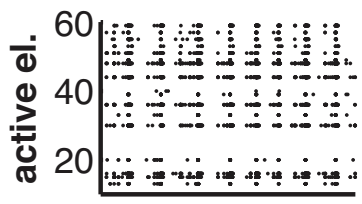
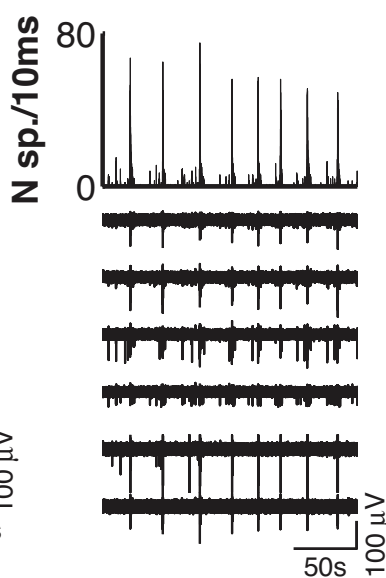

C 
that, in our case, the shunt capacitance could have been reduced by a factor of 16 upon narrowing our electrical traces to $5 \mu \mathrm{m}$, which would still not compromise the fabrication process.

The challenge of shunt capacitance for gold ultramicroelectrodes emphasizes the benefit of low impedance PEDOT:PSS coating. Packaging (e.g. gluing of culture chambers on in vitro MEAs) can also increase the impedance of microelectrodes (McDonald et al., 2017). Since PEDOT:PSS deposition is performed later in the fabrication process and includes electrochemical cleaning, this problem is entirely avoided.

In our biological experiments, we have overall demonstrated that the number of active (or recording) PEDOT:PSS ultramicroelectrodes is smaller than in conventional MEAs but still adequate for capturing the synchronized network activity emerging spontaneously in neuronal networks developing ex vivo (Figures 5A,B). The $200 \mu \mathrm{m}$ spacing between electrodes allowed us to keep cells naturally distributed in culture without the need for enforcing a patterning on their substrate adhesion, and made cell guidance not pivotal in contrast to previous contributions to the literature (Santoro et al., 2013). For this reason, we feel we might have avoided the alteration in the neurites growth pattern (Hai et al., 2009) and, as a result, obtained a more naturalistic in vitro network connectivity.

This is also supported by the average number of spikes, detected with single PEDOT:PSS ultramicroelectrodes, as it was tendentially lower than with conventional MEA electrodes. A reduction could be expected due to the electrode selectivity, suggested by our results: as PEDOT:PSS ultramicroelectrodes often detected signals from a single neuronal unit, in contrast to the typical multiunit recordings of conventional MEA electrodes. Therefore, our results seem to favor PEDOT:PSS ultramicroelectrodes as a high-selectivity mean of recording neural signals, with reduced needs for spike sorting. Of course, only future tests in vivo will conclusively establish whether a clear advantage for a less ambiguous spike sorting might be achieved with the ultramicroelectrodes. If confirmed, recorded data of such new experiments might require lower sampling frequencies, as an analysis of the spike shapes would not be strictly required, leading as a side effect to a much lower volume of data produced for each experimental session.

Signal amplitudes detected with PEDOT:PSS ultramicroelectrodes were comparable with those detected with conventional electrodes, meanwhile, a recent experiment (Shmoel et al., 2016) showed that gold ultramicroelectrodes make possible the recording of signals with amplitudes reaching several $\mathrm{mV}$ (approx. 2\% of reported neurons had amplitudes $>1 \mathrm{mV}$, with only 2/695 neurons above $2 \mathrm{mV}$ ). These positive results suggest a promising potential for these kind of 3D electrodes, indicating the directions for further improvement of technology, e.g., the neuron-microelectrode junction.

It has been already shown (Fendyur et al., 2011) that rat hippocampal cells are able to engulf micro protruding electrodes with size about 1-2 $\mu \mathrm{m}$, creating tight contacts, required for successful signal detection. However, such a systematic study did not cover the important aspect of how long and how stable is a similar detection over time. Then, by our results we offer additional evidences, suggesting that PEDOT:PSS ultramicroelectrodes are capable of recording under stable conditions the typical synchronized activity, developing ex vivo in neuronal networks over 4 weeks in vitro. Indeed, the averaged spike amplitudes were highly stable throughout the recording sessions. Even considering the capability to measure intracellular-like action potentials with protruding nanowires (Robinson et al., 2012; Xie et al., 2012; Dipalo et al., 2017; Abbott et al., 2019; Desbiolles et al., 2019), a key feature and the main advantage of microelectrode arrays (i.e. long-term noninvasive recordings) is not achieved by the nanowires. Also with a quantitative analysis of the number of penetrating nanowires, it had been shown (Aalipour et al., 2014) that the lipid bilayer and the actin cytoskeleton act as barriers to accessing the cell cytosol, so that a (chemical) poration might be still insufficient to increase long-term access to cells.

We emphasize that additional knowledge is required to achieve reliable intracellular-like recordings in mammalian cell types and, ultimately, in vivo (Spira et al., 2018). Intimate contact and surface recognition are necessary for achieving an effective high seal resistance and a low junctional membrane resistance. We recommend correlation of local surface chemistry (e.g., by X-ray photoelectron spectroscopy) with ultrastructural and molecular biological behavior (e.g., by immunochemical staining and FIB tomography). It will be in fact very important to distinguish between the macroscopic and microscopic properties, such as the different interaction with PEDOT:PSS of molecules (e.g., PEI) used for increasing cell adherence to the larger insulating area (e.g., silicon nitride).

\section{CONCLUSION}

In this paper, we presented and experimentally validated a novel type of MEAs that, to our knowledge, represent currently the best-reported combination of small electrode size and low electrical impedance, under the overall perspectives of highly parallel, non-invasive, intracellular-like recordings by extracellular MEAs. The fabrication we described is simpler than what is reported in the literature, relying only on contact lithography and avoiding electron beam lithography or advanced nanofabrication of nanowire electrodes.

The present study finally demonstrates that the presence of PEDOT:PSS ultramicroelectrodes did not alter neuronal network biological properties in vitro. The coating by a conducting polymer significantly improved the quality of extracellular recordings, when compared to uncoated ultramicroelectrodes. We conclude that ultramicroelectrodes offer further perspectives in improving the neuron-electrode interface, while enhancing the quality and sensitivity of MEAs recordings and thus representing a promising tool for our study of neuronal networks. 


\section{DATA AVAILABILITY STATEMENT}

The datasets generated for this study are available on request to the corresponding authors.

\section{ETHICS STATEMENT}

The animal study was reviewed and approved by Animal Ethics Committee of the University of Antwerp.

\section{AUTHOR CONTRIBUTIONS}

Conceived and designed the research: RS and MG Performed the experiments, fabricated the devices: AM, PJ, CB, $\mathrm{KG}, \mathrm{GH}, \mathrm{BS}$, and RS. Wrote the manuscript: AM, PJ, RS, and MG.

\section{REFERENCES}

Aalipour, A., Xu, A. M., Leal-Ortiz, S., Garner, C. C., and Melosh, N. A. (2014) Plasma membrane and actin cytoskeleton as synergistic barriers to nanowire cell penetration. Langmuir 30, 12362-12367. doi: 10.1021/la502273f

Abbott, J., Ye, T., Krenek, K., Gertner, R. S., Ban, S., Kim, Y., et al. (2019). A nanoelectrode array for obtaining intracellular recordings from thousands of connected neurons. Nat. Biomed. Eng. 4, 232-241. doi: 10.1038/s41551-0190455-7

Almquist, B. D., and Melosh, N. A. (2010). Fusion of biomimetic stealth probes into lipid bilayer cores. Proc. Natl. Acad. Sci. U.S.A. 107, 5815-5820. doi: 10 1073/pnas.0909250107

Angle, M. R., Wang, A., Thomas, A., Schaefer, A. T., and Melosh, N. A. (2014) Penetration of cell membranes and synthetic lipid bilayers by nanoprobes Biophys. J. 107, 2091-2100. doi: 10.1016/j.bpj.2014.09.023

Becchetti, A., Gullo, F., Bruno, G., Dossi, E., Lecchi, M., and Wanke, E. (2012) Exact distinction of excitatory and inhibitory neurons in neural networks: a study with GFP-GAD67 neurons optically and electrophysiologically recognized on multielectrode arrays. Front. Neural Circ. 6:63. doi: 10.3389/fncir 2012.00063

Buitenweg, J. R., Rutten, W. L. C., and Marani, E. (2002). Modeled channel distributions explain extracellular recordings from cultured neurons sealed to microelectrodes. IEEE Trans. Biomed. Eng. 49, 1580-1590. doi: 10.1109/TBME 2002.805555

Chung, T., Wang, J. Q., Wang, J., Cao, B., Li, Y., and Pang, S. W. (2015) Electrode modifications to lower electrode impedance and improve neura signal recording sensitivity. J. Neural Eng. 12:056018. doi: 10.1088/1741-2560/ $12 / 5 / 056018$

Desbiolles, B. X. E., de Coulon, E., Bertsch, A., Rohr, S., and Renaud, P. (2019). Intracellular recording of cardiomyocyte action potentials with nanopatterned volcano-shaped microelectrode arrays. Nano Lett. 19, 6173-6181. doi: 10.1021/ acs.nanolett.9b02209

Dipalo, M., Amin, H., Lovato, L., Moia, F., Caprettini, V., Messina, G. C., et al. (2017). Intracellular and extracellular recording of spontaneous action potentials in mammalian neurons and cardiac cells with 3D plasmonic nanoelectrodes. Nano Lett. 17, 3932-3939. doi: 10.1021/acs.nanolett.7b01523

Fendyur, A., Mazurski, N., Shappir, J., and Spira, M. E. (2011). Formation of essential ultrastructural interface between cultured hippocampal cells and gold mushroom-shaped MEA- Toward IN-CELL recordings from vertebrate neurons. Front. Neuroeng. 4:14. doi: 10.3389/fneng.2011.00014

Franks, W., Schenker, I., Schmutz, P., and Hierlemann, A. (2005). Impedance characterization and modeling of electrodes for biomedical applications. IEEE Trans. Biomed. Eng. 52, 1295-1302. doi: 10.1109/TBME.2005.847523

Gerwig, R., Fuchsberger, K., Schroeppel, B., Link, G. S., Heusel, G., Kraushaar, U., et al. (2012). PEDOT-CNT composite microelectrodes for recording

\section{FUNDING}

We thanks the European Commission in the 7th Framework Program (project "BRAINLEAP", Grant No. 306502), and the Bundesministerium für Bildung und Forschung ("DREPHOS” project, Grant No. 031L0059A) for financial support.

\section{ACKNOWLEDGMENTS}

We are grateful to Micha E. Spira, Martin Stelzle, Claus Burkhardt, Luc Gentet, Stefano Ferraina, and Günther Zeck for discussions, Angelika Stumpf, Andrea Herzog, Manuel Martina, and Sebastian Röhler for microfabrication support.

and electrostimulation applications: fabrication, morphology, and electrical properties. Front. Neuroeng. 5:8. doi: 10.3389/fneng.2012.00008

Guo, L. (2019). On neural recording using nanoprotrusion electrodes. J. Neural Eng. 17:016017. doi: 10.1088/1741-2552/ab51de

Hai, A., Kamber, D., Malkinson, G., Erez, H., Mazurski, N., Shappir, J., et al. (2009). Changing gears from chemical adhesion of cells to flat substrata toward engulfment of micro-protrusions by active mechanisms. J. Neural Eng. 6:066009. doi: 10.1088/1741-2560/6/6/066009

Hai, A., Shappir, J., and Spira, M. E. (2010a). In-cell recordings by extracellular microelectrodes. Nat. Methods 7, 200-202. doi: 10.1038/nmeth.1420

Hai, A., Shappir, J., and Spira, M. E. (2010b). Long-term, multisite, parallel, incell recording and stimulation by an array of extracellular microelectrodes. J. Neurophysiol. 104, 559-568. doi: 10.1152/jn.00265.2010

Humphrey, D. R., and Schmidt, E. M. (1990). "Extracellular single-unit recording methods," in Neurophysiological Techniques, II, eds A. A. Boulton, G. B. Baker and C. H. Vanderwolf (New Jersey: Humana Press), 1-64. doi: 10.1385/089603-185-3:1

Jones, P., Barthold, C., Beer, M., Burkhardt, C., Gutohrlein, K., Heusel, G., et al (2016). In vitro and in vivo probes with mushroom-shaped microelectrodes - tools for in-cell electrophysiology. Front. Neurosci. 10:76. doi: 10.3389/conf. fnins.2016.93.00076

Lin, Z. C., Xie, C., Osakada, Y., Cui, Y., and Cui, B. (2014). iridium oxide nanotube electrodes for highly sensitive and prolonged intracellular measurement of action potentials. Nat. Commun. 5:3206. doi: 10.1038/NCOMMS4206

Ludwig, K. A., Langhals, N. B., Joseph, M. D., Richardson-Burns, S. M., Hendricks, J. L., and Kipke, D. R. (2011). Poly(3,4-ethylenedioxythiophene) (PEDOT) polymer coatings facilitate smaller neural recording electrodes. J. Neural Eng. 8:014001. doi: 10.1088/1741-2560/8/1/014001

Mahmud, M., Pulizzi, R., Vasilaki, E., and Giugliano, M. (2014). QSpike tools: a generic framework for parallel batch preprocessing of extracellular neuronal signals recorded by substrate microelectrode arrays. Front. Neuroinform. 8:26. doi: 10.3389/fninf.2014.00026

Marblestone, A. H., Zamft, B. M., Maguire, Y. G., Shapiro, M. G., Cybulski, T. R., Glaser, J. I., et al. (2013). Physical principles for scalable neural recording. Front. Comput. Neurosci. 7:137. doi: 10.3389/fncom.2013.00137

McDonald, M., Monaco, A., Vahidpour, F., Haenen, K., Giugliano, M., and Nesladek, M. (2017). Diamond microelectrode arrays for in vitro neuronal recordings. MRS Commun. 7, 683-690. doi: 10.1557/mrc.2017.62

Navajas, J., Barsakcioglu, D. Y., Eftekhar, A., Jackson, A., Constandinou, T. G., and Quian Quiroga, R. (2014). Minimum requirements for accurate and efficient real-time on-chip spike sorting. J. Neurosci. Methods 230, 51-64. doi: 10.1016/j. jneumeth.2014.04.018

Neto, J. P., Baião, P., Lopes, G., Frazão, J., Nogueira, J., Fortunato, E., et al. (2018). Does impedance matter when recording spikes with polytrodes? Front. Neurosci. 12:715. doi: 10.3389/fnins.2018.00715 
Ojovan, S. M., Rabieh, N., Shmoel, N., Erez, H., Maydan, E., Cohen, A., et al. (2015). A feasibility study of multi-site, intracellular recordings from mammalian neurons by extracellular gold mushroom-shaped microelectrodes. Sci. Rep. 5:14100. doi: 10.1038/srep14100

Piccirillo, A., and Gobbi, A. L. (1990). Physical-electrical properties of silicon nitride deposited by PECVD on III-V semiconductors. J. Electrochem. Soc. 137:3910. doi: 10.1149/1.2086326

Piela, B., and Wrona, P. K. (1995). Capacitance of the gold electrode in $0.5 \mathrm{M}$ H2SO4 solution: a.c. impedance studies. J. Electroanal. Chem. 388, 69-79. doi: 10.1016/0022-0728(94)03848-W

Plummer, J. D., Deal, M. D., and Griffin, P. B. (2000). Silicon VLSI Technology: Fundamentals, Practice, and Modeling. Upper Saddle River, NJ:: Prentice Hall.

Qing, Q., Jiang, Z., Xu, L., Gao, R., Mai, L., and Lieber, C. M. (2014). Freestanding kinked nanowire transistor probes for targeted intracellular recording in three dimensions. Nat. Nanotechnol. 9, 142-147. doi: 10.1038/nnano. 2013.273

Quiroga, R. Q., Nadasdy, Z., and Ben-Shaul, Y. (2004). Unsupervised spike detection and sorting with wavelets and superparamagnetic clustering. Neural Comput. 16, 1661-1687. doi: 10.1162/089976604774201631

Robinson, D. A. (1968). The electrical properties of metal microelectrodes. Proc. IEEE 56, 1065-1071. doi: 10.1109/PROC.1968.6458

Robinson, J. T., Jorgolli, M., Shalek, A. K., Yoon, M.-H., Gertner, R. S., and Park, H. (2012). Vertical nanowire electrode arrays as a scalable platform for intracellular interfacing to neuronal circuits. Nat. Nanotechnol. 7, 180-184. doi: 10.1038/nnano.2011.249

Sahalianov, I., Singh, S. K., Tybrandt, K., Berggren, M., and Zozoulenko, I. (2019). The intrinsic volumetric capacitance of conducting polymers: pseudocapacitors or double-layer supercapacitors? RSC Adv. 9, 42498-42508. doi: 10. 1039/C9RA10250G

Santoro, F., Schnitker, J., Panaitov, G., and Andreas, O. (2013). On chip guidance and recording of cardiomyocytes with $3 \mathrm{D}$ mushroom-shaped electrodes. Nano Lett. 13, 5379-5384. doi: 10.1021/nl402901y

Scott, K. M., Du, J., Lester, H. A., and Masmanidis, S. C. (2012). Variability of acute extracellular action potential measurements with multisite silicon probes. J. Neurosci. Methods 211, 22-30. doi: 10.1016/j.jneumeth.2012. 08.005

Shmoel, N., Rabieh, N., Ojovan, S. M., Erez, H., Maydan, E., and Spira, M. E. (2016). Multisite electrophysiological recordings by self-assembled loose-patchlike junctions between cultured hippocampal neurons and mushroom-shaped microelectrodes. Scie. Rep. 6:27110. doi: 10.1038/srep27110

Spira, M. E., and Hai, A. (2013). Multi-electrode array technologies for neuroscience and cardiology. Nat. Nanotechnol. 8, 83-94. doi: 10.1038/nnano. 2012.265
Spira, M. E., Shmoel, N., Huang, S.-H. M., and Erez, H. (2018). Multisite attenuated intracellular recordings by extracellular multielectrode arrays, a perspective. Front. Neurosci. 12:212. doi: 10.3389/fnins.2018.00212

Trasatti, S., and Petrii, O. A. (1992). Real surface area measurements in electrochemistry. J. Electroanal. Chem. 327, 353-376. doi: 10.1016/00220728(92)80162-W

Viswam, V., Obien, M. E. J., Franke, F., Frey, U., and Hierlemann, A. (2019). Optimal electrode size for multi-scale extracellular-potential recording from neuronal assemblies. Front. Neurosci. 13:385. doi: 10.3389/fnins.2019.00385

Weidlich, S., Krause, K. J., Schnitker, J., Wolfrum, B., and Offenhäusser, A. (2017). MEAs and 3D nanoelectrodes: electrodeposition as tool for a precisely controlled nanofabrication. Nanotechnology 28:095302. doi: 10.1088/13616528/aa57b5

Weir, K., Blanquie, O., Kilb, W., Luhmann, H. J., and Sinning, A. (2014). Comparison of spike parameters from optically identified GABAergic and glutamatergic neurons in sparse cortical cultures. Front. Cell. Neurosci. 8:460. doi: 10.3389/fncel.2014.00460

Wellman, S. M., Eles, J. R., Ludwig, K. A., Seymour, J. P., Michelson, N. J., McFadden, W. E., et al. (2018). A materials roadmap to functional neural interface design. Adv. Funct. Mater. 28:1701269. doi: 10.1002/adfm.201701269

Wesche, M., Hüske, M., Yakushenko, A., Brüggemann, D., Mayer, D., Offenhäusser, A., et al. (2012). A nanoporous alumina microelectrode array for functional cell-chip coupling. Nanotechnology 23:495303. doi: 10.1088/0957-4484/23/49/ 495303

Xie, C., Lin, Z., Hanson, L., Cui, Y., and Cui, B. (2012). Intracellular recording of action potentials by nanopillar electroporation. Nat. Nanotechnol. 7, 185-190. doi: $10.1038 /$ nnano.2012.8

Conflict of Interest: RS is employed by NMI TT GmbH, which sells microelectrode arrays.

The remaining authors declare no competing financial interests. $\mathrm{CB}$ and $\mathrm{KG}$ contributed to this work while affiliated with NMI at the University of Tübingen, and are currently employed by joimax $\mathrm{GmbH}$ and Bosch Sensortec $\mathrm{GmbH}$, respectively.

Copyright (c) 2020 Jones, Moskalyuk, Barthold, Gutöhrlein, Heusel, Schröppel, Samba and Giugliano. This is an open-access article distributed under the terms of the Creative Commons Attribution License (CC BY). The use, distribution or reproduction in other forums is permitted, provided the original author(s) and the copyright owner(s) are credited and that the original publication in this journal is cited, in accordance with accepted academic practice. No use, distribution or reproduction is permitted which does not comply with these terms. 